\title{
Clinical Trials Corner Issue 7(2)
}

Piyush K. Agarwal ${ }^{\mathrm{a}, 1}$ and Cora N. Sternberg ${ }^{\mathrm{b}, 1}$

${ }^{a}$ The University of Chicago, Chicago, IL, USA

${ }^{\mathrm{b}}$ Englander Institute for Precision Medicine, Weill Cornell Medicine, New York, NY, USA

Received 1 May 2021

Accepted 9 May 2021

Pre-press 19 May 2021

Published 25 May 2021

Dear Readers,

In this issue, we highlight 2 important phase III trials that were presented at ASCO GU in 2021, one with the antibody drug conjugate Enfortumab Vedotin, in advanced disease and one in the adjuvant setting. We also highlight an adjuvant trial that has been completed and another one that is actively recruiting patients. The trials answer important clinical questions about adjuvant immunotherapy after cystectomy in patients with muscle invasive urothelial cancer (MIUC). In the future, please reach out to us directly in order to highlight any specific clinical trials at pkagarwal@uchicago.edu or cns9006@med.cornell.edu and/or at BLC@ iospress.com.

\section{Sincerely,}

Piyush K. Agarwal, MD

Associate Editor, Bladder Cancer

Director, Bladder Cancer Program

The University of Chicago

Chicago, Illinois
Cora N. Sternberg, MD, FACP

Associate Editor, Bladder Cancer

Clinical Director, Englander Institute for Precision Medicine

Weill Cornell Medicine

New York, New York

Study Title: A Study to Evaluate Enfortumab Vedotin Versus (vs) Chemotherapy in Subjects With Previously Treated Locally Advanced or Metastatic Urothelial Cancer (EV-301) ${ }^{1}$

\section{Clinicaltrials.gov identifier: NCT03474107}

Sponsor: Astellas Pharma Global Development

Enrollment: 608 patients

Conclusions: Enfortumab vedotin significantly prolonged survival as compared with standard chemotherapy (CT) in patients with locally advanced or metastatic urothelial carcinoma who had previously received platinumbased treatment and a PD-1 or PD-L1 inhibitor. (Funded by Astellas Pharma US and Seagen; EV-301)

Rationale: Patients with advanced urothelial carcinoma have poor overall survival when recurring after platinum-containing chemotherapy and programmed cell death protein 1 (PD-1) or programmed death ligand 1 (PD-L1) inhibitor treatment. More novel therapies are needed. 
Study Design: This was an international open-label, phase 3 trial of enfortumab vedotin, an antibody drug conjugate that targets Nectin-4 and carries a Monomethyl Auristatin E (MMAE) Payload, a microtubuledisrupting agent, as compared to investigator's chemotherapy of choice in patients with locally advanced or metastatic urothelial carcinoma who had previously received platinum-containing chemotherapy and had disease progression during or after treatment with a PD-1 or PD-L1 inhibitor. Patients were randomly assigned in a 1:1 ratio to receive enfortumab vedotin or docetaxel, paclitaxel, or vinflunine.

Endpoints: The primary end point was overall survival (OS).

Results: 301 patients were assigned to receive enfortumab vedotin and 307 to receive chemotherapy. As of July 15,2020 , a total of 301 deaths had occurred (134 in the enfortumab vedotin group and 167 in the chemotherapy group). At the prespecified interim analysis, the median follow-up was 11.1 months. OS was longer in the enfortumab vedotin group than in the chemotherapy group (median 12.88 vs. 8.97 months; hazard ratio for death, $0.70 ; 95 \%$ confidence interval $[\mathrm{CI}], 0.56$ to $0.89 ; \mathrm{P}=0.001$ ). Progression-free survival(PFS) was also longer in the enfortumab vedotin group than in the chemotherapy group (median 5.55 vs. 3.71 months; HR for progression or death, $0.62 ; 95 \% \mathrm{CI}, 0.51$ to $0.75 ; \mathrm{P}<0.001$ ). The study was halted by the IDMC at this analysis and it was recommended to the sponsor to allow crossover of patients on chemotherapy at PD to enfortumab vedotin.

Comments: The FDA approved enfortumab vedotin for treatment of patients with locally advanced or metastatic UC previously treated with a PD-(L)1 inhibitor and platinum-containing CT in the (neo)adjuvant, locally advanced, or metastatic setting. A second antibody drug conjugate Sacituzumab Govitecan, that targets Targets Trop-2, with a SN-38 Payload, the active metabolite of irinotecan, was also recently approved for locally advanced or metastatic UC previously treated with a PD-L1 inhibitor and either platinum ineligible or previously treated with platinum-containing CT in the (neo)adjuvant, locally advanced, or metastatic setting.

\section{Study Title: An Investigational Immuno-therapy Study of Nivolumab, Compared to Placebo, in Patients With Bladder or Upper Urinary Tract Cancer, Following Surgery to Remove the Cancer (CheckMate 274) $)^{2}$}

Clinicaltrials.gov identifier: NCT02632409

Sponsor: Bristol-Myers Squibb

\section{Enrollment: 709}

Rationale: Radical surgical resection is the standard of care for patients with MIUC. Adjuvant therapy after radical surgery is not currently recommended for patients who have received neoadjuvant therapy... For patients who have not had neoadjuvant cisplatin-based CT, adjuvant cisplatin-based CT may be offered but the level of evidence is low. Nivolumab, a PD-1 immune checkpoint inhibitor, is approved as monotherapy for treatment of platinum-resistant metastatic UC. Until this trial, no immunotherapy has shown efficacy as adjuvant therapy in patients with MIUC at high risk for recurrence after radical surgery.

Study Design: CheckMate 274 is a phase 3, randomized, double-blind, multicenter study of adjuvant nivolumab versus placebo in patients with high-risk MIUC. Inclusion criteria were: Patients with ypT2-ypT4a or ypN+ MIUC who had neoadjuvant cisplatin-based CT, or pT3-pT4a or pN+ MIUC without prior neoadjuvant cisplatinbased CT, and not eligible for or refused adjuvant cisplatin-based CT; radical surgery performed within the past 120 days and disease-free status within 4 weeks of dosing. Patients were randomized 1:1 to nivolumb IV 240 mg every 2 weeks vs. placebo IV every 2 weeks x 1 year. Stratification factors included: PD-L1 status $(<1 \%$ vs $\geq 1 \%$ ), prior neoadjuvant cisplatin-based chemotherapy and nodal status. 
Endpoints: The primary endpoints were disease free survival in the intent to treat (ITT) population and DFS in all randomized patients with tumor PD-L1 $\geq 1 \%$.

Results: Adjuvant nivolumab significantly improved DFS in patients with high-risk MIUC after radical surgery, both in the ITT and PD-L1 $\geq 1 \%$ populations. In the ITT population, the median DFS with nivolumab was 21.0 (17.1-33.4) months vs $10.9(8.3-13.9)$ months, HR, $0.70(98.31 \%$ CI, 0.54-0.89) in patients treated with placebo $(\mathrm{p}<0.001)$. In the PD-L1 $\geq 1 \%$ population, median DFS was NR $(22.0-\mathrm{NE})$ vs 10.8 (5.7-21.2) months, HR, $0.53(98.87 \%$ CI, 0.34-0.84), respectively $(\mathrm{p}<0.001)$. No deterioration in health related quality of life, as measured by change in EORTC QLQ-C30 global health status score, was observed with nivolumab vs placebo.

Comments: Nivolumab is the first systemic immunotherapy to demonstrate a statistically significant and clinically meaningful improvement in outcomes when administered as adjuvant therapy to patients with MIUC. The FDA has granted priority review, although the overall survival data are not yet mature. These results may well support nivolumab monotherapy as a new standard of care in the adjuvant setting for patients with highrisk MIUC after radical surgery, irrespective of PD-L1 status and prior neoadjuvant chemotherapy. The results differ from what was found in the adjuvant atezolizumab trial highlighted below.

\section{Study Title: A Study of Atezolizumab Versus Observation as Adjuvant Therapy in Participants With High-Risk Muscle-Invasive Urothelial Carcinoma (UC) After Surgical Resection (IMvigor010) ${ }^{3}$}

\section{Clinicaltrials.gov identifier: NCT02450331}

Sponsor: F Hoffmann-La Roche/Genentech

\section{Enrollment: 809}

Rationale: As mentioned earlier in the nivolumab aduvant trial, despite standard curative-intent treatment with neoadjuvant cisplatin-based chemotherapy, followed by radical surgery in eligible patients, muscle-invasive urothelial carcinoma has a high recurrence rate and there is not enough clincal trial evidence supporting adjuvant therapy. This trial aimed to evaluate atezolizumab as adjuvant therapy in patients with high-risk MIUC.

Study Design: IMvigor010 was a multicenter international open-label, randomized, phase 3 trial for patients after radical cystectomy or nephroureterectomy with lymph node dissection. Patients had ypT2-4a or ypN+ tumors following neoadjuvant chemotherapy or $\mathrm{pT} 3-4 \mathrm{a}$ or $\mathrm{pN}+$ tumors if no neoadjuvant chemotherapy was received. Patients not treated with neoadjuvant chemotherapy were required to be cisplatin ineligible or declined cisplatin-based adjuvant chemotherapy. Patients were randomized (1:1) to atezolizumab anti PDL1 immunotherapy IV every 3 weeks for 1 year vs. observation. Randomization was stratified by previous neoadjuvant chemotherapy use, number of lymph nodes resected, pathological nodal status, tumor stage, and PD-L1 expression on tumor-infiltrating immune cells.

Endpoints: The primary endpoint was disease-free survival in the ITT population.

Results: 406 patients were assigned to the atezolizumab group and 403 were assigned to observation. Median follow-up was 21.9 months (IQR 13.2-29.8). Median DFS was 19.4 months (95\% CI 15.9-24.8) with atezolizumab and 16.6 months (11.2-24.8) with observation (stratified hazard ratio 0.89 [95\% CI 0.74-1.08]; $\mathrm{p}=0.24)$.

Comments: IMvigor010 was the first-completed phase 3 adjuvant study to evaluate the role of a checkpoint inhibitor in MIUC. The trial did not meet its primary endpoint of improved DFS in the atezolizumab group compared to observation. Atezolizumab was generally tolerable, with no new safety signals; however, higher frequencies of adverse events leading to discontinuation were reported than in metastatic urothelial carcinoma 
studies. These data do not support the use of adjuvant atezolizumab therapy in MIUC. The results differ from those in the nivolumab study. There were more patients who discontinued therapy in this study and the reasons for the differences between the two trials require further exploration.

Study Title: Study Title: Testing MK-3475 (Pembrolizumab) After Surgery for Localized MuscleInvasive Bladder Cancer and Locally Advanced Urothelial Cancer (AMBASSADOR) ${ }^{4}$

\section{Clinicaltrials.gov identifier: NCT03244384}

Sponsor: National Cancer Institute (NCI)

Enrollment: 739 planned

Rationale: As mentioned in the two adjuvant trials above, there is not enough clinical trial evidence supporting adjuvant therapy in high risk MIUC. Similar to the two trials described above, this trial aims to evaluate pembrolizumab, a PD-1 immune checkpoint inhibitor, approved as monotherapy for treatment of platinumresistant metastatic UC and platinum ineligible patients as well as in NMIBC patient who have failed BCG, as adjuvant therapy in patients with high-risk MIUC.

Study Design: Eligible patients for the trial have high-risk muscle-invasive bladder cancer or upper tract urothelial carcinoma (UTUC). They must have undergone a cystectomy or nephrectomy within 16 weeks, have pT2-4aNx or $\mathrm{pTxN}+$ disease after having undergone neoadjuvant chemotherapy. Patients can have pT3$4 \mathrm{Nx}$ or $\mathrm{pN}+$ disease post surgery with no chemotherapy. Patients are stratified by PD-L1 positivity, receipt of prior neoadjuvant chemotherapy, and pathologic stage pT2/3/4aN0 vs. pT4bNx or N1-3 disease. They are then randomized to receive $200 \mathrm{mg}$ of pembrolizumab every three weeks for 12 months or to observation. Healthcare-related quality of life outcomes will also be measured.

End Points: The primary objectives of this study are to determine overall survival (OS) and DFS

Results: Still accruing

Comments: It is hoped that this study will finish its accrual after presentation of the positive adjuvant Nivolumab trial.

\section{CONFLICT OF INTEREST}

\section{Cora N. Sternberg}

Consultant: Pfizer, Merck Ga, AstraZeneca, Astellas Pharma, Sanofi-Genzyme, Roche/Genentech, Medscape, Immunomedics, Clovis Oncology, BMS, Janssen, UroToday, MSD, Foundation Medicine

\section{Piyush K. Agarwal}

Advisory Board (unpaid): AstraZeneca; Advisory Board (paid): AURA, Verity

\section{References:}

1. Powles T et al, N Engl J Med, 2021 Mar 25;384(12):1125-1135. doi: 10.1056/NEJMoa2035807.Epub 2021 Feb 12.

2. Bajorin D et al, ASCO GU, Abstract Number 391

3. Bellmunt J et al, Lancet Oncol. 2021 Apr;22(4):525-537. doi: 10.1016/S1470-2045(21)00004-8. Epub 2021 Mar 12.

4. Apolo A, ASCO GU 2019, abstract 